\title{
Analysis of Country-wide Internet Outages Caused by Censorship
}

\author{
Alberto Dainotti \\ University of Napoli Federico II \\ alberto@unina.it \\ Kimberly C. Claffy \\ CAIDA/UCSD \\ kc@caida.org \\ Michele Russo \\ University of Napoli Federico II \\ mic.russo83@gmail.com
}

Claudio Squarcella

Roma Tre University

squarcel@dia.uniroma3.it

\author{
Marco Chiesa \\ Roma Tre University \\ chiesa@dia.uniroma3.it \\ Antonio Pescapé \\ University of Napoli Federico II \\ pescape@unina.it
}

\begin{abstract}
In the first months of 2011, Internet communications were disrupted in several North African countries in response to civilian protests and threats of civil war. In this paper we analyze episodes of these disruptions in two countries: Egypt and Libya. Our analysis relies on multiple sources of large-scale data already available to academic researchers: BGP interdomain routing control plane data; unsolicited data plane traffic to unassigned address space; active macroscopic traceroute measurements; RIR delegation files; and MaxMind's geolocation database. We used the latter two data sets to determine which IP address ranges were allocated to entities within each country, and then mapped these IP addresses of interest to BGP-announced address ranges (prefixes) and origin ASes using publicly available BGP data repositories in the U.S. and Europe. We then analyzed observable activity related to these sets of prefixes and ASes throughout the censorship episodes. Using both control plane and data plane data sets in combination allowed us to narrow down which forms of Internet access disruption were implemented in a given region over time. Among other insights, we detected what we believe were Libya's attempts to test firewallbased blocking before they executed more aggressive BGP-based disconnection. Our methodology could be used, and automated, to detect outages or similar macroscopically disruptive events in other geographic or topological regions.
\end{abstract}

\section{Categories and Subject Descriptors}

C.2.3 [Network Operations]: Network Monitoring;

C.2.5 [Local and Wide-Area Networks]: Internet

\section{General Terms}

Measurement, Security

Permission to make digital or hard copies of all or part of this work for personal or classroom use is granted without fee provided that copies are not made or distributed for profit or commercial advantage and that copies bear this notice and the full citation on the first page. To copy otherwise, to republish, to post on servers or to redistribute to lists, requires prior specific permission and/or a fee.

IMC'11, November 2-4, 2011, Berlin, Germany.

Copyright 2011 ACM 978-1-4503-1013-0/11/11 ...\$10.00.

\section{Keywords}

Outages, Connectivity Disruption, Censorship, Darknet, Network Telescope, Internet Background Radiation

\section{INTRODUCTION}

On the evening of January 27, 2011 Egypt-a population of 80 million, including 23 million Internet users [41]—vanished from the Internet. The Egyptian government ordered a complete Internet shutdown amidst popular anti-government protests calling for the resignation of Egyptian President Hosni Mubarak. The order followed reports on the previous day (25 January) of blocked access to Twitter [24], although an Egyptian government official denied blocking any social media web sites [48]. In addition to mainstream media reporting of traffic disruption [49], several commercial Internet measurement companies posted technical data analyses on their blogs $[16,32,50]$. The heavy-handed attempt to block communications in the country did not quell the protests, and may have even increased the number of people in the streets; protests intensified and continued even after Internet connectivity was restored five days later. Under political pressure from inside and outside Egypt, President Hosni Mubarak resigned, turning command over to the military on February 11.

Four days later, similar protests erupted in Libya, calling for an end to the Gaddafi regime. On February 17 major protests took place across the country [44], and that evening YouTube became inaccessible from Libya [25]. On the night of February 18 (Friday) the government imposed an "Internet curfew", blocking all Internet access until morning (08:01 local time), and repeating it the next day (Saturday) [16, 32, 63]. In the following days, Libyan traffic to popular sites like Google increased steadily [26] until Internet access was disabled again, this time for nearly four days. Figure 1 presents a brief chronology of the events.

Wide-scale Internet service disruptions are nothing new $[12,15$, 57,66-69], even politically-motivated interference with Internet access in order to hinder anti-government organization [3,8]. But the scale, duration, coverage, and violent context of Egypt's and Libya's disruptions has brought the Internet "kill switch" issue into new critical light.

In this paper we analyze the Internet disruptions that took place in Egypt and Libya in early 2011. These two events are of historical as well as scientific interest. Access to publicly available Internet measurement data that cover the outage intervals allows empirical 


\section{Egypt}

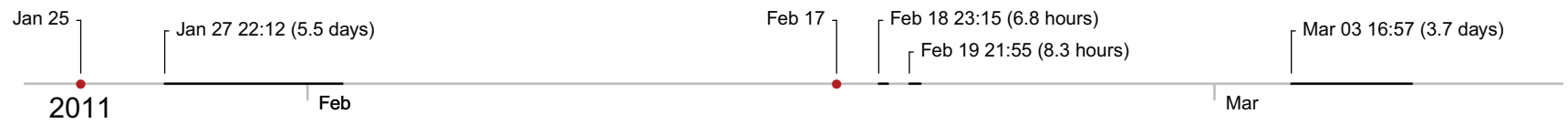

Figure 1: Timeline of Internet disruptions described in the paper. Times in figure are UTC (Egypt and Libya are UTC+2). The pair of red dots indicate the start of major political protests in the respective countries.

study of what it takes to bring down an entire country's communications infrastructure, which has security relevance for every nation in the world. We were also able to observe surprisingly noticeable effects of such large scale censorship on ongoing global measurement activities, suggesting how similar events could be detected and/or documented in the future. Our analysis relies on multiple measurements and vantage points:

- Traffic to unassigned address space, specifically data collected from the UCSD network telescope [7], reveal changes in background Internet traffic from the affected regions.

- BGP data from RouteViews [59] and RIPE NCC's Routing Information Service (RIS) [6] provide a view of BGP activity during the events.

- Active traceroute probing from Ark [2] reveals forward path information and can reveal additional types of filtering, e.g. firewalling, not observable via BGP, as well as help calibrate BGP observations.

We focus on two episodes of Internet disruption, although the same global data sources and our proposed methodology could illuminate the study of similar events in other countries. The main contributions of this paper are:

* We document a rich view of the disruptions using three types of measurement data sources.

* We demonstrate how to use unsolicited background traffic to identify country-level blocking events.

* We report previously unknown details of each event, including the use of packet filtering as well as BGP route withdrawals to effect the disruption.

* We sketch a general methodology for the analysis of some types of of disruptions that combines multiple measurement data sources available to researchers.

* Our methodology and findings can form the basis for automated early-warning detection systems for Internet service suppression events.

The rest of this paper is organized as follows. Section 2 gives technical background on network disruption, limiting its focus to the paper's scope. Section 3 summarizes related work. Section 4 describes our measurement and analysis methodology. Section 5 presents the results of our analysis. Section 6 discusses our findings and concludes the paper.

\section{BACKGROUND}

Disabling Internet access is an extreme form of Internet censorship in which a population's Internet access is blocked completely, a coarse but technically more straightforward approach than the selective blocking used in most Internet censorship regimes. It can be implemented by simply powering down or physically disconnecting critical equipment, although this approach typically requires physical co-location with the communications equipment, which may be spread over a wide area. A more flexible approach is to use software to disrupt either the routing or packet forwarding mechanisms.

Routing disruption. While forwarding is the mechanism that advances packets from source to destination, the routing mechanism determines which parts of the network are reachable and how. On the Internet, global routing is coordinated at the level of Autonomous Systems (ASes) - administratively distinct parts of the network - using BGP as the interdomain routing protocol. Routers exchange BGP information (BGP updates) regarding which destination addresses they can reach, and continually update their forwarding tables to use the best available path to each contiguous block of destination addresses. ${ }^{1}$ Disabling the routing process on critical routers or suppressing BGP information transmission can effectively render large parts of a network unreachable.

Packet filtering. Packet filtering intervenes in the normal packet forwarding mechanism to block (i.e., not forward) packets matching given criteria. Today packet filtering of varying complexity is a standard security feature of network equipment from switches to dedicated firewalls. ${ }^{2}$

Disabling a network's connectivity to the Internet through BGP routing disruption is easily detectable, since it entails changes in the global routing state of the network, i.e., in the control plane. Previously advertised prefixes must be withdrawn or re-advertised with different properties in order to change global routing behavior. Detecting packet filtering is harder; it requires either active probing of the forward path, or monitoring traffic (the data plane) from the affected parts of the network for changes.

We also note that BGP-based traffic control and packet-filtering are only two of many layers where censorship of connectivity and content may occur. A taxonomy of blocking technologies would include a range of approaches, including physical layer disconnection, content blocking based on deep packet inspection by an ISP, DNS-based blocking or manipulation at multiple granularities, and end-client software blocking in an enterprise or household.

\section{RELATED WORK}

An Internet blackout is put into effect by a government when more selective forms of censorship are either impractical or ineffective. Governments use selective censorship to restrict Internet traffic for political, religious, and cultural reasons. Recent studies have found that Internet censorship is widespread and on the rise $[3,8]$. Unsurprisingly, there is also growing interest in analyzing the technical aspects of censorship, and methods to circumvent them.

\footnotetext{
${ }^{1}$ Destination addresses are advertised as prefixes, ranges of addresses represented as a set of fixed high-order bits and varying low-order bits. A prefixes is written as an IP address in dotted quad notation followed by a " $"$ " and the prefix length. For example, the prefix 132.239.0.0/17 represents IP addresses 132.239.0.0 to 132.239 .127 .255 .

${ }^{2}$ Because of their versatile packet filtering capabilities, firewalls can flexibly implement many types of selective censorship: address filtering, protocol filtering, and simple content filtering.
} 
In [14] Clayton et al. analyzed the keyword filtering mechanism of the "Great Firewall of China" (GFC) and found that it was possible to circumvent it by simply ignoring the forged TCP packets with the reset flag set sent by the firewall. In [21], Crandall et al. disproved the notion that the GFC is a firewall strictly operating at the border of China's Internet. They showed that filtering can occur at different hops past the Chinese border (cases of up to 13 IP address hops were observed), suggesting that filtering happens at the AS level. They also proposed ConceptDoppler, a system for monitoring which keywords are filtered over time. In more recent work on censorship in China [64], Xueyang Xu et al. explored the AS-level topology of China's network and probed the firewall to find the locations of filtering devices. They found that two major ISPs in China had different approaches to the placement of filtering devices, either decentralized or in the backbone. Results also showed that most of the filtering happened in "border ASes", that is, ASes that peer with foreign networks. In [53] Skoric et al. provided a detailed analysis of how social media platforms (e.g., Facebook), as well as traditional media were used to organize a student protest against censorship in Singapore. They found that activists used social media to engage rather than circumvent traditional media stakeholders, in order to amplify their impact. Trying to prevent such amplification was a likely motivation for the Internet blackouts we analyzed in this study.

The scientific study of Internet interdomain routing has a longer and richer history (thus far) than the study of Internet censorship. Academic researchers have used BGP data to support scientific study of Internet dynamics for over a decade, including Labovitz et al.'s studies in the late 1990's of several root causes of high levels Internet routing instability $[35,36]$. They traced a large fraction of unnecessary ("pathological") BGP update volume to router vendor software implementation decisions, and their research convinced router vendors to modify BGP parameters, decreasing the volume of Internet routing updates they observed a year later by an order of magnitude. More recently researchers have explored the use of spatial and temporal correlations among the behaviors of BGP prefixes to detect and hopefully predict instabilities $[23,30]$.

In [51] Sahoo et al. used simulations to estimate BGP recovery time for networks with a range of topological characteristics undergoing large-scale failure scenarios, such as those caused by disastrous natural or man-made events. They showed that topological properties, especially the node degree distribution, can significantly affect recovery time. In [38] $\mathrm{Li}$ and Brooks proposed an Internet seismograph to consistently measure the impact of disruptive events such as large-scale power outages, undersea cable cuts and worms, to enable quantitative comparison of the impact of different events. Other researchers have also used BGP monitoring infrastructure to analyze the impact of these types of disruptive events on the routing system [19,20,37]. In [61] Tao Wan and Van Oorschot used data from the RouteViews project [59] during Google's outage in 2005, showing what was probably a malicious BGP advertisement of a Google network prefix by another AS.

Several analysis and real-time BGP monitoring tools have also been developed. In [56] Teoh et al. presented BGP Eye, a tool for root-cause analysis and visualization of BGP anomalies based on clustering of BGP updates into events that are compared across different border routers. The visualization helps to show events that may affect prefix reachability and hijacking. In [29] Katz-Bassett et al. introduced Hubble, a prototype system that operated continuously for a couple of years to find Internet reachability problems in real-time, specifically for situations where routes exist to a destination but packets are unable to reach the destination. While the Hubble system detected unreachability of prefixes, it did not aggregate results beyond a single AS. Dan Massey's group at Colorado State is maintaining BGPMon, a real-time BGP routing instrumentation system [65] designed to scalably monitor BGP updates and routing tables from many BGP routers simultaneously, while also allowing user-defined real-time "notification" feeds of specific subsets of the data.

Regarding the specific blackouts we describe in this paper, several commercial Internet measurement companies posted technical data analyses on their blogs during and following the outages [11,16-18,31-34,60]. Most of them analyzed flow-level traffic data or BGP updates seen by their routers, providing timely news and technical insights, although omitting specifics about the proprietary data and analysis methodologies used.

Our work combines multiple different data sources available to academic researchers to analyze in detail the characteristics of two large-scale censorship episodes that occurred early this year. None of the data analysis studies described above were designed to detect phenomena at a country granularity, nor to integrate traffic, topology, and routing data, although their techniques could complement the ones we present in this paper to improve detection of country-wide network disruptions. Moreover, while BGP data has often been used to monitor network infrastructure dynamics during outages, we are not aware of any previous work using unsolicited (sometimes called darknet or telescope) traffic to monitor such outages, although we proposed it in March 2011 [10]. Our approach facilitated the discovery of important technical aspects of the outages not previously reported: the combination of packet filtering techniques with BGP disruption, and disruption of satellite Internet connectivity.

\section{DATA SOURCES AND METHODOLOGY}

\subsection{Internet Geography}

To properly observe the effects of a blackout in a given country, we first need to identify which Internet numbering resources are under the control of, or related to, those countries. In this section we explain how we used geolocation data to select the relevant set of IP addresses, BGP prefixes, and AS numbers to monitor for visibility into Egypt and Libya during the blackout intervals.

\subsubsection{IP Addresses}

Five Regional Internet Registries (RIRs) manage the allocation and registration of Internet resources (IP prefixes and autonomous system numbers) to entities within five distinct regions of the world, and publish lists of the Internet resources they delegate, which include the country hosting the headquarters of the receiving entity. Egypt and Libya are in the AfriNIC (Africa's) region [1]. The first row of Table 1 lists the number of IPv4 addresses delegated to Egypt and Libya by AfriNIC. Additionally, IP addresses nominally allocated to a different country may be used within Egypt and Libya if a foreign ISP provides Internet access in those countries. The second row of Table 1 lists the IP addresses geolocated in Egypt and Libya according to the MaxMind GeoLite Country database [40]. We used these two sources of data to construct the list of IP address ranges (prefixes) that geolocated to Egypt and Libya.

Although there are accuracy issues in all geolocation databases $[27,46,52]$, at a country granularity these databases almost always agree with (sometimes because they are based on) the RIR-delegation file information. Countries with either low Internet penetration or a small population (Libya has both) may only have few IPs officially RIR-delegated to them, in which case IP geolocation database information can provide useful additional information. Satellite con- 


\begin{tabular}{lcc}
\hline & Egypt & Libya \\
\hline AfriNIC delegated IPs & $5,762,816$ & 299,008 \\
MaxMind GeoLite IPs & $5,710,240$ & 307,225 \\
\hline
\end{tabular}

Table 1: IPv4 address space delegated to Egypt (as of January 24, 2011) and Libya (as of February 15, 2011) by AfriNIC (top half) as well as additional IPv4 address ranges associated with the two countries based on MaxMind GeoLite database (as of January 2011).

nectivity, which at least one ISP uses to serve Libya, is another source of IP geolocation discrepancy.

\subsubsection{AS Numbers and BGP-announced prefixes}

Once we had derived a set of IP address ranges to monitor, we mapped these to BGP-announced prefixes and ASes announcing those prefixes, using a database constructed from publicly available BGP data from RouteViews and RIPE NCC RIS [6,59] for the week preceding the outages, up to and including the first day of the outage. The allocated address ranges might not map precisely to a BGP prefix boundary, since ASes may implement complex routing policies to accomplish traffic engineering and other business objectives, which may involve splitting BGP prefixes into smaller chunks, or aggregating prefixes into larger chunks to reduce routing table sizes. Thus, different views of the routing system (BGP monitors) may have different BGP prefixes covering a given set of IP addresses. Once we gather BGP events within the time window we want to observe, we compute the set of covering prefixes $P$ for address space $S$ as follows:

- We look up the address space in the BGP database described above, to find an exactly matching BGP prefix;

- We find all the more specific (strict subset, longer) prefixes of this prefix;

- if the two previous steps yielded no prefix, we retrieve the longest BGP prefix entirely containing the address space $S$.

For each AS we show results only for the IP ranges or BGP prefixes that are solely related to the country under analysis, e.g., traffic whose source addresses are included in prefixes announced by that AS and are geolocated or delegated to the country under analysis.

\subsection{BGP Data}

BGP routing changes can rapidly induce global effects, including coarse-grained filtering that may be indistinguishable from complete physical disconnection of infrastructure. Using BGP data in conjunction with data-plane measurements such as traceroute or traffic flows can yield a rich understanding of the type of censorship strategy being used.

The two main sources of BGP updates used throughout our analysis are the already mentioned Route Views Project [59] and RIPE NCC's Routing Information Service (RIS) [6], respectively maintained by University of Oregon and RIPE NCC. Both services rely on routers that establish BGP peering sessions with many ISPs around the world. The available data reveal a broad and global though necessarily incomplete view of BGP connectivity over time, at a announced-prefix granularity. We analyzed this data at the finest possible time granularity - BGP updates - to detect and isolate events observed during the outages. However, BGP updates only provide information about changes in routing state. Each route collector also periodically dumps a snapshot of its entire control plane table, called a $R I B$, containing all known routing information related to prefixes that are reachable at that point in time. We used these periodic dumps in conjunction with the fine-grained updates to track a precise view of prefix reachability over the duration of the outage intervals.

Each source of data also has a graphical tool to query for specific prefixes, BGPlay [58] for Route Views and BGPviz [4] for RIS. An online service called REX [5] allows coarse-grained analysis of historical BGP events in RIS data, based on snapshots taken every 8 hours of the RIB table dumps. (Routeviews RIBs are dumped every two hours.)

To perform chronological analysis of the outages, we first identified the time window during which disconnection activity was observed, using previous reports [16, 17], BGPlay and BGPviz. We extended this window to one hour before and one hour after the main events we observed to detect possible early symptoms of the outage and late reconnection patterns. We used the last RIB table dumps from both RouteViews and RIS just before this interval as the starting point, and used BGP updates and subsequent BGP table dumps to reconstruct the routing history during the time window under examination.

For each prefix we processed the downloaded data to build a set of routing histories, one for each route collector peer (that is, an AS feeding its BGP updates to a route collector). We marked a prefix as disappeared if it was withdrawn during the blackout interval for each of the above routing histories, i.e., no longer observable from any route collector peer, and remained so for the duration of the interval. We chose the earliest of those withdrawals as the event representing the initial disconnection of the prefix. This approach provides an overview of the prefixes going down over time, as well as the first signs of disconnection for each withdrawn prefix.

We used a similar approach to study the end of the outage, focusing instead on when BGP prefixes become reachable again via announcements seen in BGP updates. BGPlay and BGPviz were also useful to visualize the transition periods, to see which prefixes were still visible as the outage information propagated and to see peers reconverge to secondary backup paths. We visualized the reconnection as well, with peers reverting to primary paths as prefixes were re-announced.

Note that the collected data is subject to uncertainty, especially regarding timing of events, so we cannot obtain a perfect understanding of BGP dynamics. BGP messages are sometimes delayed (aggregated) by routers in order to minimize control traffic. Furthermore, router clocks are not necessarily synchronized, so one cannot be sure of the exact interleaving sequence of events occurring at different routers. However, empirical analysis of data coming from different collectors generally shows good correlation in terms of time values. While imperfect, our methodology provides a consistent way to approximate the timing of BGP withdrawals during an outage.

\subsection{Darknet Traffic}

Unsolicited one-way Internet traffic, also called Internet background radiation $(I B R)$ [45], has been used for years to study malicious activity on the Internet including worms, DoS attacks, and scanning address space looking for vulnerabilities to exploit. Such a vast number of computers generate such background radiation, mostly unbeknownst to their legitimate users, that the resulting traffic aggregate has proven a useful source of data for observing characteristics of the malware itself $[22,42,43]$ not revealed by other types of data.

Researchers observe IBR traffic using network telescopes, often called darknets if the IP addresses are not being used by devices. We collected and analyzed traffic arriving at the UCSD network telescope [7], which observes a mostly unassigned $/ 8$, that is, 
1/256th of the entire IPv4 address space. We used the same IP-AS databases described in Section 4.1 to determine the levels of unsolicited traffic throughout the outages in Egypt and Libya. Although the unsolicited traffic from these countries exhibited a typical diurnal pattern, sudden changes in the packet rate suggest start and end times of several of the outages (see Figure 2).

There are three primary causes of IBR traffic: (i) backscatter from spoofed denial-of-service (DoS) attacks, (ii) scans, or (iii) bugs and misconfiguration [45]. Different types of IBR traffic induce separate sources of packet rate dynamics, which can heavily affect the amount of traffic observed from a specific country to the network telescope. Our methodology identifies and separates these sources of IBR-induced dynamics to avoid misinterpreting them.

Packets associated with denial-of-service attacks represent a special category, because they cause substantial packet rate variation, especially for countries with few IP addresses such as Egypt and Libya. A DoS attack attempts to overwhelm a victim with traffic or transaction requests in order to reduce or prevent his ability to serve legitimate requests. When the source IP addresses in attacking packets are randomly spoofed, the response packets (e.g. $S Y N$ $A C K$ TCP packets in reply to $S Y N$ s from the attacker) are sent back to the spoofed addresses, producing backscatter, which will be captured by telescopes [43] that happen to contain (and thus observe traffic to) those spoofed addresses. To identify and characterize these attacks we used the methodology in [43], separating potential backscatter packet flows (i.e. TCP packets with SYN-ACK or RST flags on, ICMP echo replies) by sender (potential victims of the attack), then classifying any such flows above predefined volume or duration thresholds as backscatter reflecting a denial-of-service attack. Of course, DoS attacks to a country in civil unrest may themselves be of interest; we did notice attacks on some government web sites approximately when the protests began and a short time before the outage (see Sections 5.1.3 and 5.2.3.)

Automated (e.g. from worms) or manually initiated random scanning of address space in search of victims is another component of IBR [22,42]. On 21 November 2008, the amount of unsolicited traffic grew dramatically with the advent of the Conficker worm [9], which widely infected Windows hosts and actively scanned for hosts to infect on TCP port 445. Sufficiently pervasive network scanning such as done by Conficker reveals surprisingly detailed insights into global Internet behavior. In particular, geolocation of all IP source addresses of such scanning packets makes it easy to detect country-wide outages [10], since an entire country disappears from this category of traffic. We identified Conficker scanning traffic by selecting TCP SYN packets with destination port 445 and packet size 48 bytes.

Misconfiguration of systems can also induce IBR traffic, for example by setting the wrong IP address on a DNS or proxy server. Bugs in network applications and router firmware and software e.g., getting byte ordering wrong, can assign incorrect network addresses to a device, triggering unsolicited traffic in response.

We further analyzed the unsolicited traffic by Autonomous System (AS) coming from within each of the two countries, revealing AS-specific behavior. IP address space that has been withdrawn from the global BGP routing table will not be able to receive traffic from the Internet default-free zone anymore, but may be able to successfully send outbound traffic in the absence of packet filtering. Analysis of background radiation, especially Conficker-like scanning, reveals some of this leaked traffic.

\subsection{Active forward path probing}

We made limited use of active measurements taken during the outages in Egypt and Libya, toward address space in these coun- tries. The measurements consisted both of ad-hoc measurements using standard ping and traceroute, and of structured global IPv4 address probing from CAIDA's IPv4 Routed /24 Topology Dataset [28] collected on CAIDA's Ark infrastructure [2]. We used the IPv4 topology data set to observe surprising 2-way connectivity surviving the outage intervals that span more then a day. This data does not provide sufficient granularity to analyze the shorter outages due to Ark's low probing rate (one cycle of /24 IPv4 prefixes in about 3 days) and the relatively few IP prefixes in each country.

\section{ANALYSIS}

In this section we present our analysis of the Internet blackouts from the perspectives of the control plane (BGP data) and the data plane (traffic and traceroute data). Section 5.1 discusses the outage in Egypt; Section 5.2 discusses the several disconnections in Libya. To prevent unintentional harm to those involved in these episodes of censorship or their circumvention, we have anonymized most AS numbers in this paper as described in Appendix A.

\subsection{Egypt}

\subsubsection{Overview}

According to Greg Mahlknecht's cable map web site [39], which might have dated information, Egypt's Internet infrastructure is dominated by state ownership, consisting primarily of a few large players with international connectivity through the major submarine cable systems that run through the Suez canal. Most, if not all, submarine fiber-optic circuits are backhauled to the Ramses Exchange [62], which is not only the main connection facility for the Egyptian-government-controlled telecommunications provider, but also the location of the largest Internet exchange point in North Africa or the Middle East. Both the small number of parties involved in international connectivity, and the physical connectivity under control of the state telecommunications provider, facilitate manipulation of the system by a state actor, as shown by the events described below.

Renesys reported that on January 27, around 22:34:00 GMT, they observed the "virtually simultaneous withdrawal of all routes to Egyptian networks in the Internet's global routing table” [16]. The packet rate of unsolicited traffic from Egypt seen by the UCSD telescope (Figure 2) suddenly decreased at almost the same time, on January 27 around 22:32:00 GMT. In terms of BGP, the methodology explained in Section 4 identifies the outage as a sequence of routing events between approximately 22:12:00 GMT and 22:34:00 GMT. The outage lasted for more than five days, during which more active BGP IPv4 prefixes in Egypt were withdrawn. In Figure 3 each step represents a set of IPv4 prefixes at the point in time when they first disappeared from the network.

The UCSD darknet traffic returned to packet rates comparable to those preceding the outage at 10:00:00 GMT on February 2. The unsolicited traffic level from Egypt to the telescope was roughly consistent with our BGP analysis, which found that the first set of re-announcements of Egyptian connectivity after the crisis occurred around 09:29:31 GMT. Figure 4 shows the BGP connectivity reappearing.

\subsubsection{Outages in detail}

BGP data reveals a dramatic drop in reachability for many Egyptian IPv4 prefixes during the outage. It is not obvious which event should be considered the first sign of the outage. The leftmost end of the graph in Figure 3 shows 2928 IPv4 prefixes visible via BGP at 20:00:00 GMT. A noticeable loss of connectivity is first seen by RouteViews and RIS route collectors on January 27 at 20:24:11 
GMT, related to $15 \mathrm{IPv} 4$ prefixes routed by EgAS2. ${ }^{3}$. Further losses of BGP connectivity are visible in the next two hours, summing up to 236 withdrawn IPv4 prefixes. The biggest disruption then appears as an almost vertical drop in Figure 3, with the initial step at 22:12:26 GMT, after which roughly 2500 prefixes disappear within a 20 minute interval. At 23:30:00 GMT only 176 prefixes remain visible.

Figure 5 shows the same sequence of events separated by the six main Egyptian ASes. Although the image seems to suggest a time sequence for the interleaving BGP withdrawals, we can make no safe assumption on the chronology of underlying decisions.

Contrary to IPv4 prefixes, there was no major change in visibility for IPv6 prefixes. Of the six IPv6 prefixes in AfriNIC's delegated file, only one is seen in RIS and this prefix of length /32 is announced by IntAS1, a major international carrier. This prefix stayed visible during the outage, as did all its more specific prefixes seen in RIS (20/48s announced by EgAS4 and 1 /48 by EgAS6).

Figure 6 shows a breakdown of the traffic observed by the UCSD network telescope in three categories: conficker, backscatter, and other. Conficker refers to TCP SYN packets with destination port 445 and packet size 48 bytes. While we assume that these packets are generated by systems infected by the Conficker worm scanning for new victims, we cannot be absolutely certain, although our inferences are valid if the majority of packets satisfy this assumption. The most important implication of the assumption is that the source IP addresses are not spoofed; if they were, the geolocation mapping would be meaningless.

The backscatter category of traffic requires careful treatment. When an attacker uses fake source IP addresses in a denial-ofservice attack targeting a victim in the address space we are trying to observe, backscatter traffic from the victim IP addresses can increase suddenly and dramatically, jeopardizing our inferences about background traffic levels coming from this address space. So our methodology must identify and filter out this backscatter traffic.

The other category represents all other packets composing the background radiation [45] captured by the network telescope: worms, generic scanning and probing activity, misconfigurations, etc.

Figure 6 reveals the diurnal patterns of activity in Conficker traf-

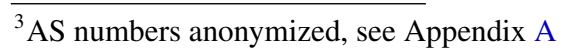

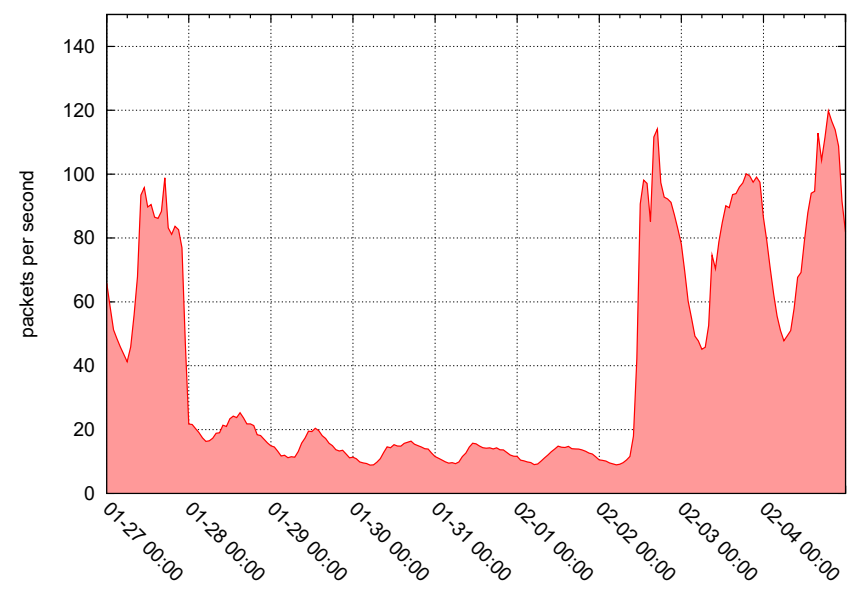

Figure 2: Unsolicited packets from IPs geolocated in Egypt to UCSD's network telescope. The two dramatic changes in the packet rate respectively match the withdrawals and reannoucements of BGP routes to Egyptian networks. fic, which are typical of (infected) PCs that are not kept on 24 hours per day. Conficker traffic is the dominant component, and stays partially alive even during the outage, for two reasons: (i) some prefixes are still visible via BGP and (ii) outbound connectivity still works for some networks. For a given network, BGP withdrawals are a consequence of either propagation of a withdrawal from elsewhere in the network, or a data-plane failure immediately adjacent to the router. In the former case the network is unreachable from the outside world, but may still be able to send packets in the outbound direction. In the same figure, the backscatter traffic has some spikes related to a couple of denial-of-service attacks which we discuss in Section 5.1.3.

The other category of traffic in Figure 6 is most interesting: soon after the Egyptian networks are again BGP-reachable, the packet rate of this traffic grows much higher than before the outage. By analyzing traffic from the entire Internet reaching the UCSD darknet, we found that a large UDP/TCP scan targeted toward a specific service was conducted from thousands of hosts all around the world. ${ }^{4}$ The diurnal pattern suggests this traffic was a coordinated scan operated by a botnet, which started on January 31st (based on global traffic to the telescope) and lasted several days. It looks like the Egyptian hosts infected by the botnet lost communication with the botnet control channel during the outage, but after BGP connectivity returned, they started to participate in the coordinated scan. The interesting insight is that scanning activities under botnet control cannot operate in the absence of bidirectional connectivity (since the bots cannot communicate with their controller) but random scans from worm-infected hosts still do, and are still visible by the telescope when the senders are not BGP-reachable but still connected to the network. Such gaps between what the telescope can see globally versus from a specific country can help define criteria for the automated detection and classification of such events.

More than 3165 IPv4 prefixes are delegated to Egypt and managed by 51 ASes. In order to sketch per-AS observations by the network telescope, we classify the traffic observed from Egyptian IP addresses by the AS responsible for ("originating") the IP addresses. Figure 7 shows the packet rate of traffic observed by the telescope from two major ASes in Egypt: EgStateAS, and EgAS4. EgStateAS is the largest Egyptian Internet service provider.

Figure 5 shows that many of the prefixes announced by EgStateAS via BGP were withdrawn on or shortly after January 27 . A small set

\footnotetext{
${ }^{4}$ The UDP packets sent during this scan have specific properties that allow us to recognize them, but details are beyond the scope of the paper
}

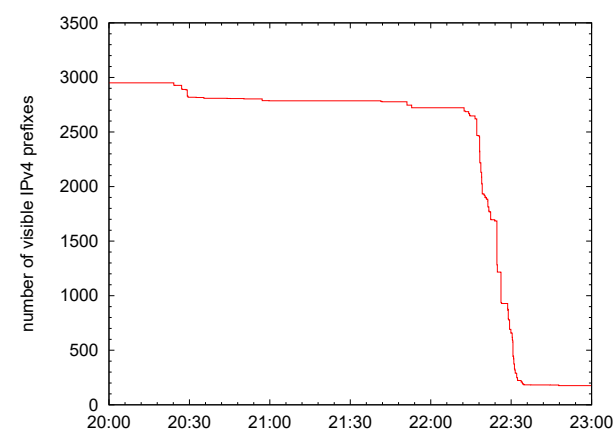

Figure 3: Disconnection of Egyptian IPv4 prefixes via BGP during the outage on January 27, based on data from RouteViews and RIPE NCC's RIS. For each disconnected prefix, the red line drops down at the instant in which a lasting (i.e., not temporarily fluctuating) BGP withdrawal is first observed. 


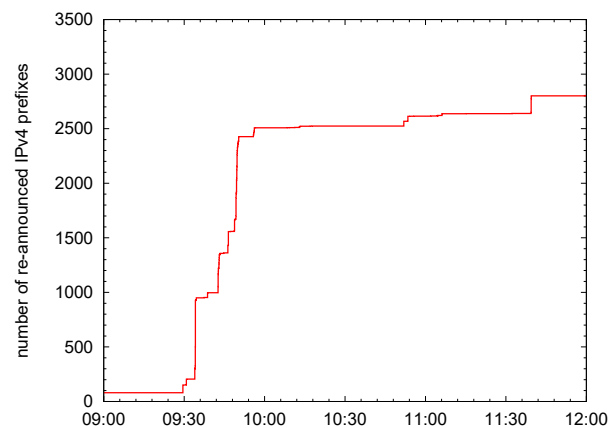

Figure 4: Re-announcement of Egyptian IPv4 prefixes via BGP at the end of the outage on February 2, based on data from RouteViews and RIPE NCC's RIS. For each re-announced prefix, the red line goes up at the instant in which a stable BGP announcement is first detected.

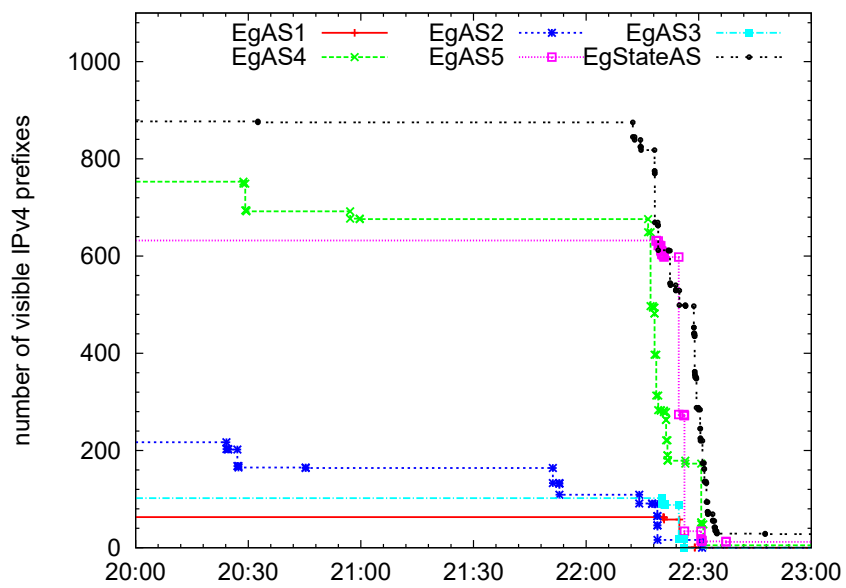

Figure 5: Visibility of main Egyptian Autonomous Systems via BGP during the outage on January 27 (based on data from RouteViews and RIPE NCC's RIS). Each AS is plotted independently; as in Figure 3, each line drops down at the instant in which a lasting (i.e., not temporarily fluctuating) BGP withdrawal is first observed.

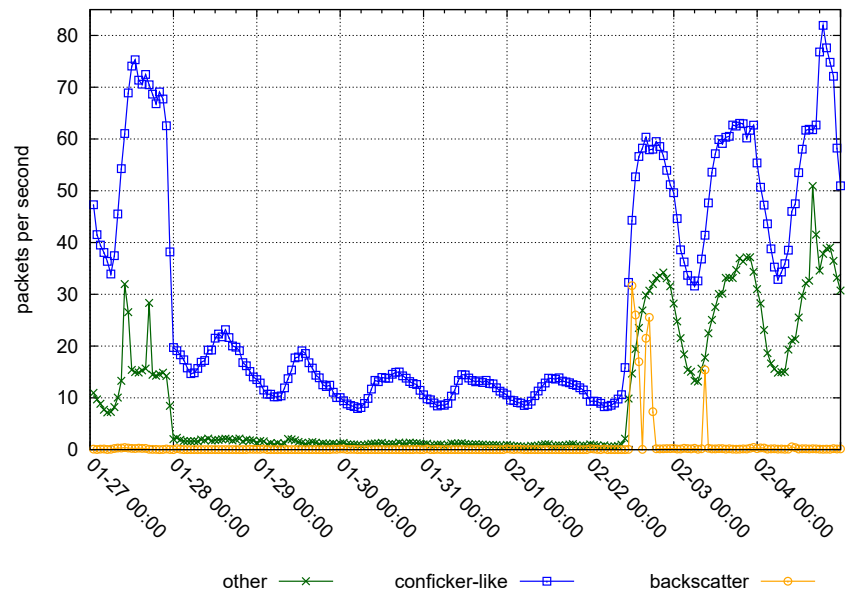

Figure 6: Categories of unsolicited packets from IPs geolocated in Egypt to UCSD's network telescope: other, conficker-like, backscatter. Spikes in backscatter traffic reflect large denial-of-service attacks against hosts in Egypt. of IPv4 prefixes remained visible during the outage, including some not announced in the previous months. For this reason we still observe darknet traffic coming from this AS, whereas the prefixes of EgAS4 were all withdrawn. A closer look at EgStateAS reveals that several of the visible IPv4 prefixes were reachable through IntAS2 or IntAS3, either because they were already served by those two Autonomous Systems or they rerouted to paths using those ASes after the massive disconnection. Figures 8(a) and 8(b) illustrate this behavior, where a prefix previously using IntAS4 as its only externally visible upstream switches to IntAS2 once the outage begins.

Finally, we ran ad-hoc active measurements during the outage to some related prefixes. In particular, we sent ICMP echo requests on 1 February at 09:00:00 GMT from GARR (the Italian Research and Academic Network), the replies to which revealed that at least three IPv4 prefixes, among those announced by EgStateAS and not withdrawn during the outage, were actually reachable. Traceroute probes simultaneously issued toward the same destinations went through IntAS2.

Another interesting case is that of EgAS7. As also reported by Renesys [16], the 83 prefixes managed by this AS remained untouched for several days during the Egyptian Internet outage. There was speculation that this AS retained Internet connectivity due to its high-profile, economically-relevant customers, including the Egyptian stock exchange, the National Bank of Egypt, and the Commercial International Bank of Egypt. However, at a certain point the censorship was tightened in Egypt: we observed the withdrawals of all 83 prefixes, almost simultaneously, on Monday, January 31, 20:46:48 GMT until the end of the outage, when all the Egyptian routes were restored. Figure 9 shows a perfect match between our telescope observation of Egyptian traffic from EgAS7 and the BGP reachability of its prefixes.

Figure 10 plots reachability statistics of active measurements from CAIDA's Ark infrastructure, revealing that during the outage, $1 \%$ of measurements to IPv4 prefixes geolocated in Egypt reached a responding destination, whereas on normal days it is closer to 16-17\%. Examination of the specific IP addresses that retained bidirectional connectivity throughout the outage confirms that they all match BGP prefixes that were not withdrawn.

At the end of the outage, a steady reconnection is observed via

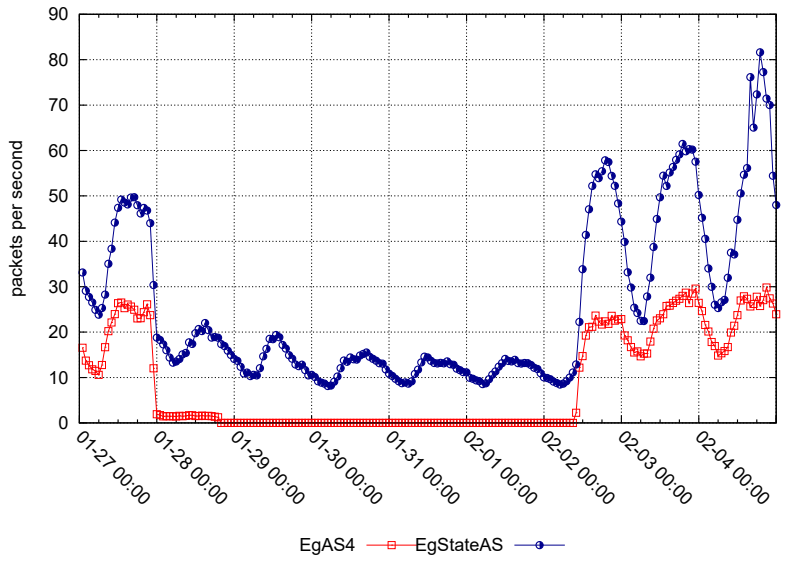

Figure 7: Unsolicited packets from IPs geolocated in Egypt to UCSD network telescope: EgAS4, EgStateAS. Traffic from EgStateAS is still significant during the outage because: (i) some prefixes remain visible; (ii) some networks probably retain outbound connectivity. The decay observable in the first days of the outage matches the progressive withdrawal of further routes. 
BGP. Figures 4 and 11 respectively show time-series of BGP announcements in aggregate and for each of the six larger ASes. Figure 11 shows each AS re-injecting sets of previously withdrawn routes, with most of them globally visible within 20 minutes. The process began with a first step at 09:29:31 GMT; by 09:56:11 GMT more than 2500 Egyptian IPv4 prefixes are back in BGP tables around the world. BGP data suggests that the key decisions on the outage were quite synchronized, and produced dramatic globally observable consequences.

\subsubsection{Denial-of-service attacks}

Analysis of the UCSD darknet traffic also allowed us to identify some denial-of-service attacks to institutional sites of the Egyptian government, which because of the timing and victims look strongly

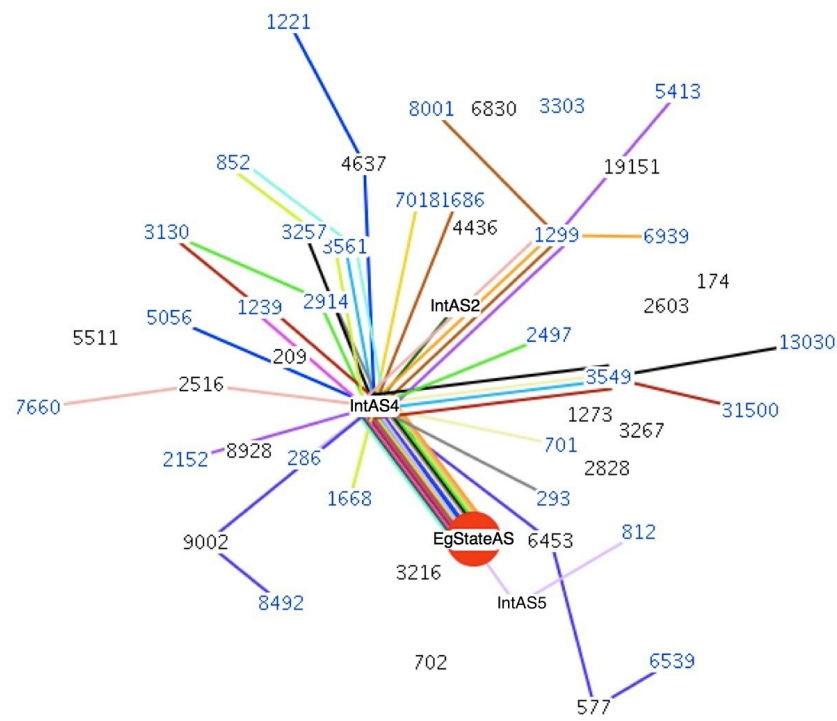

(a)

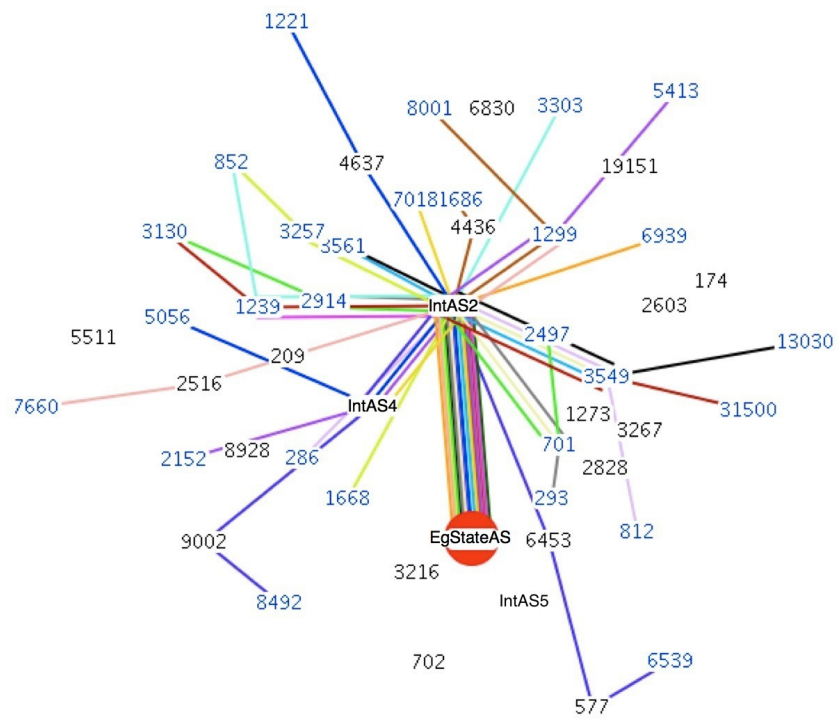

(b)

Figure 8: BGPlay snapshot showing the reachability graph of a prefix owned by EgStateAS before (a) and after (b) the outage on January 27. Each color represents the path used by an AS to reach the target prefix.

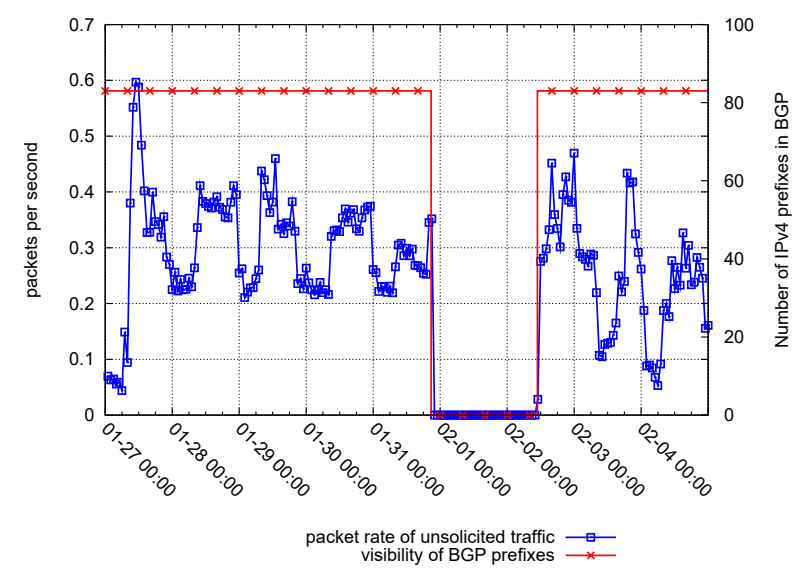

Figure 9: The case of EgAS7: a perfect match across data sources: unsolicited traffic to UCSD's network telescope vs. BGP reachability of its 83 prefixes.

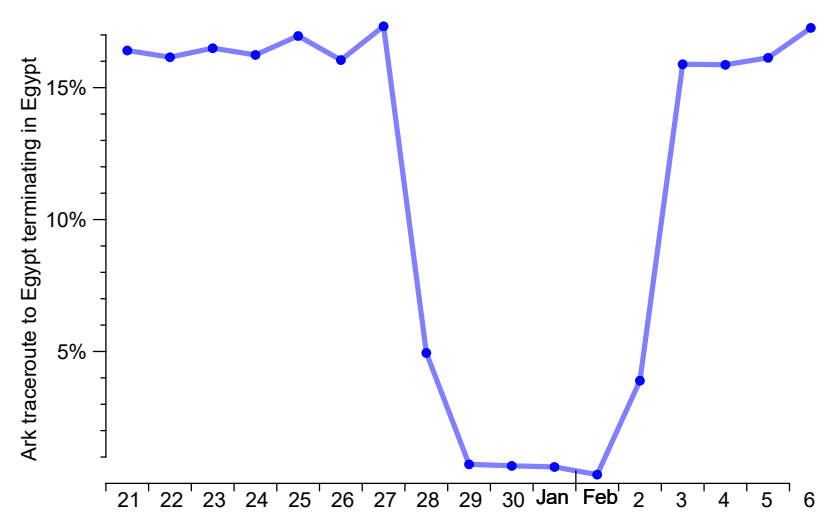

Figure 10: Fraction of Ark traceroutes that terminated (either at the destination or the last reachable hop) in Egypt. The few IP addresses that retained bi-directional connectivity (required for traceroute) throughout the outage were in BGP prefixes that were not withdrawn.

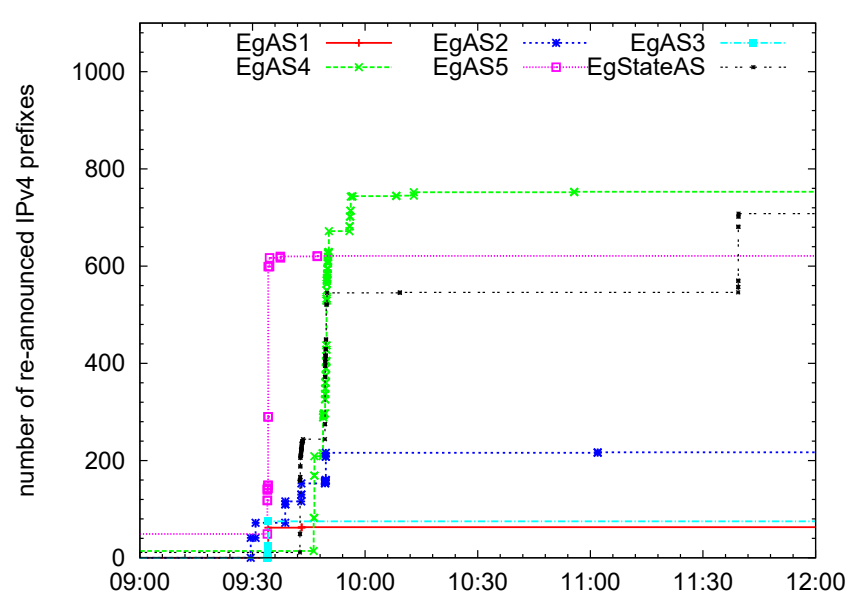

Figure 11: Reconnection of main Egyptian Autonomous Systems via BGP at the end of outage on February 2, based on data from RouteViews and RIPE NCC's RIS. Each AS is plotted independently; as in Figure 4, each line rises at the instant in which a stable BGP announcement from that AS is first observed. 


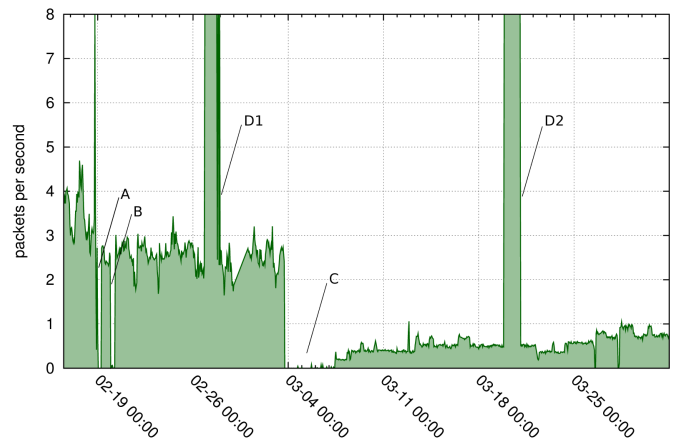

Figure 12: UCSD darknet's traffic coming from Libya. Labels A, B, C indicate the three outages. Spikes labeled D1 and D2 are due to backscatter from two denial-of-service attacks.

related to protests in the country. The web site of the Ministry of Communications (mcit.gov.eg) was attacked with a randomlyspoofed DoS attack just before the outage started, on January 26 at different times: 15:47 GMT (for 16 minutes), 16:55 GMT (17 minutes), and 21:09 GMT (53 minutes). Analysis of the backscatter traffic to the darknet allows estimation of the intensity of the attack in terms of packet rate, indicating average packet rates between $20 \mathrm{k}$ and $50 \mathrm{k}$ packets per second.

On February 2 the web site of the Egyptian Ministry of Interior (www.moiegypt.gov.eg) was targeted by two DoS attacks just after the end of the censorship from 11:05 to 13:39 GMT and from 15:08 to 17:17 GMT. The same IP address was attacked another time the day after, from 08:06 to 08:42 GMT. In this case the estimated packet rates were smaller, around $7 \mathrm{k}$ packets per second.

\subsection{Libya}

\subsubsection{Overview}

Libya's Internet infrastructure is even more prone to manipulation then Egypt's, judging from its physical structure. International connectivity is provided by only two submarine cables, both ending in Tripoli [39], and the Internet infrastructure is dominated by a single, state owned, AS. We only found two other ASes having a small presence in Libya, as described in Section 5.2.2.

In Libya three different outages in early 2011 were identified and publicly documented (Figure 1). Figure 12 shows the traffic observed by the UCSD network telescope from Libya throughout an interval encompassing the outages. The points labeled A, B and $\mathrm{C}$ indicate three different blackout episodes; points D1 and D2 refer to two denial-of-service attacks discussed in Section 5.2.3. Toward the right of the graph it is difficult to interpret what is really happening in Libya because of the civil war.

\subsubsection{Outages in detail}

The first two outages happened during two consecutive nights. Figure 13(a) shows a more detailed view of these two outages as observed by the UCSD telescope. Figure 13(b) shows BGP data over the same interval: in both cases, within a few minutes, 12 out of the $13 \mathrm{IPv} 4$ prefixes associated with IP address ranges officially delegated to Libya were withdrawn. These twelve IPv4 prefixes were announced by LyStateAS, the local telecom operator, while the remaining IPv4 prefix was managed by IntAS2. As of May 2011, there were no IPv6 prefixes in AfriNIC's delegated file for Libya. The MaxMind IP geolocation database further puts 12 noncontiguous IP ranges in Libya, all part of an encompassing IPv4 prefix announced by SatAS1, which provides satellite services in the Middle East, Asia and Africa. The covering IPv4 prefix also contained 180 IP ranges in several other countries predominantly in the Middle East. We considered this additional AS because the UCSD darknet generally observed a significant amount of unsolicited traffic coming from IPs in those 12 ranges before the first outage (about 50k packets each day). This level of background traffic indicates a population of customers using PCs likely infected by Conficker or other malware, allowing inference of network conditions. Traffic from this network also provided evidence of what happened to Libyan Internet connections based on satellite systems not managed by the local telecom provider.

Comparing Figures 13(a) and 13(b) reveals a different behavior that conflicts with previous reports [17]: the second outage was not entirely caused by BGP withdrawals. The BGP shutdown began on February 19 around 21:58.55 UTC, exactly matching the sharp decrease of darknet traffic from Libya (and in accordance with reports on Libyan traffic seen by Arbor Networks [31]) but it ended approximately one hour later, at 23:02.52. In contrast, the Internet outage as shown by the telescope data and reported by the news [17] lasted until approximately February 20 at 6:12 UTC. This finding suggests that a different disruption technique - a packet-blocking strategy apparently adopted subsequently in the third outage and recognized by the rest of the world - was already being used dur-

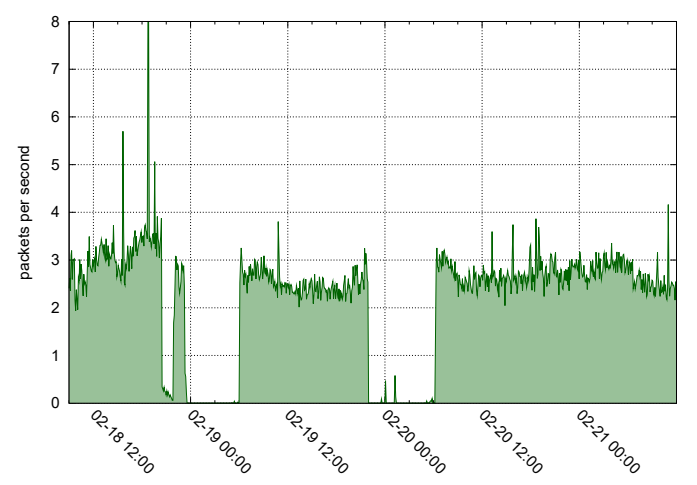

(a)

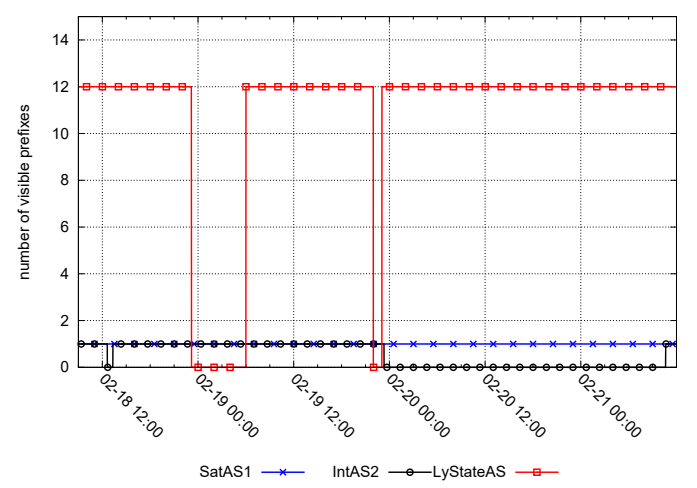

(b)

Figure 13: The first two Libyan outages: (a) unsolicited traffic to UCSD darknet coming from Libya; (b) visibility of Libyan IPv4 prefixes in BGP data from RouteViews and RIPE NCC RIS collectors. Note that the controlplane and data-plane observations of connectivity do not match, suggesting that different techniques for censorship were being used during different intervals. 
ing this second outage. The firewall configuration may have been set up during the BGP shutdown and the routes were restored once the packet blocking was put in place.

Figure 13(b) shows that the IPv4 prefix managed by SatAS1, the satellite company, was not withdrawn, which seems reasonable considering that this IPv4 prefix was managed by a company outside of Libya. But the darknet traffic from both the local telecom and SatAS1 plummeted when the two outages occurred (see Figure 14). A tiny amount of traffic still reached UCSD's darknet from SatAS1 IPs in Libya, especially during the second outage (Figure 14), suggesting that the government could have used signal jamming to disrupt the satellite service for Internet connectivity, as they did for satellite TV news and mobile communication services $[47,54]$.

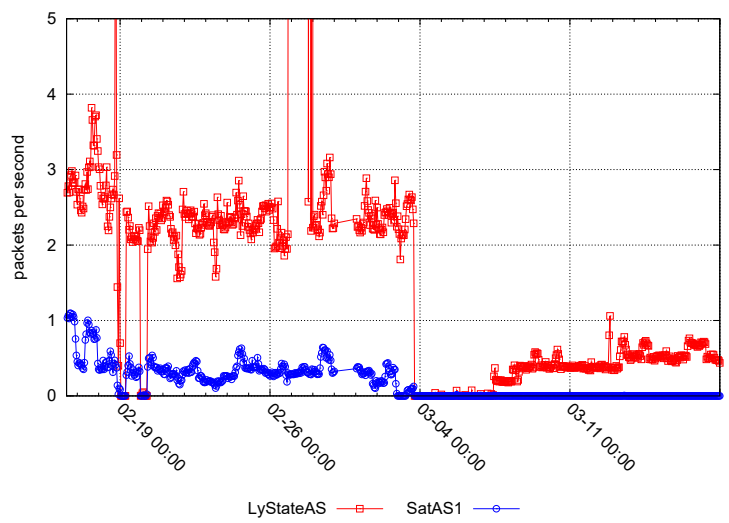

Figure 14: UCSD darknet's traffic coming from Libya: traffic from selected ASes. The connectivity of satellite-based provider SatAS1 was probably disrupted through deliberate jamming of the satellite signal.

As for IntAS2, there was not enough unsolicited traffic reaching the darknet preceding and during the outages to usefully analyze, likely due to lack of end users in this network. However, the only Libyan IPv4 prefix announced by IntAS2 was withdrawn twice: (i) on the same day of the first outage but several hours before it started (for approximately 40 minutes, from $12: 38.58$ to $12: 41.25$ UTC); (ii) approximately 10 minutes after the BGP routes of the local telecom were withdrawn in the second outage. The matching times in the latter case suggest a form of coordination or forcing the common loss of BGP connectivity. Figure 13(b) shows that the BGP disruption of the Libyan IPv4 prefix of IntAS2 lasted for about two days (from February 19 23:20.22 UTC to February 21 10:38.15 UTC), far longer than the duration of the second outage.

The third outage in Libya happened several days later. We verified, by analyzing all BGP updates collected by RouteViews and RIPE NCC RIS, that all BGP routes stayed up without interruption. However, Figure 16 shows that the darknet traffic sharply dropped at March 3 16:57:00 UTC. Perhaps not surprisingly given their earlier experimenting with different censorship techniques, the third and longest Libyan outage was not caused by BGP disruption, but by packet filtering, confirmed by other sources [18].

While probing in the Ark data was not frequent enough to see the first two Libyan outages, the third, and longer outage caused a significant drop in the fraction of reachable destinations in IPv4 prefixes geolocated in Libya, as seen in Figure 15. The remaining reachable destinations in Libya were both from wired and satellitebased ASes, showing that bidirectional connectivity for some hosts in both types of networks was possible during this longer outage, although the drop in reachable destinations suggests two-way connectivity was available for significantly fewer hosts.

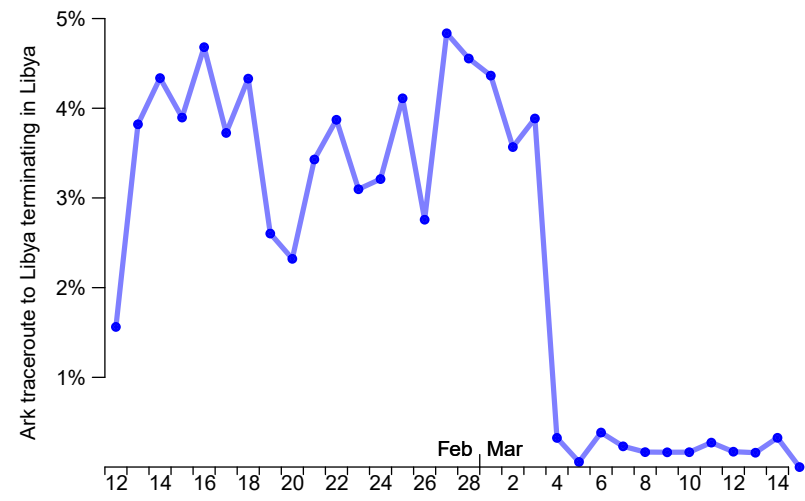

Figure 15: Fraction of Ark traceroutes that terminated (either at the destination or the last reachable hop) in Libya.

Our analysis revealed three discoveries:

- We established the potential of network telescopes to detect country-wide filtering phenomena, even phenomena that cannot be detected by monitoring BGP connectivity. The sharp decrease in traffic shown in Figure 16 suggests that a simple change point detection algorithm would automatically raise an alert in this case, similar to how others used sharp drops in observed BGP announcements.

- We confirmed that packet filtering techniques for censorship were used, because we still had visibility of a few packets from a few subnets, suggesting that perhaps the regime wanted to preserve connectivity for some sites.

- We discovered that packet filtering techniques were also used for previous outages that were reported as BGP-only disruptions. Moreover, we captured a retrospective of what happened, also explaining the short gap (February 18 from 20:24 to 21:57 UTC) in traffic visible on the UCSD network telescope (Figure 13(a)) a few hours before the first outage. This short drop in traffic was also visible in data published by others [31], but was never discussed. We verified (Figure 13(b)) that all the BGP routes were up during this gap in observed traffic, which suggests that Libya was already testing firewall blocking during this interval. The fact that the first two out-

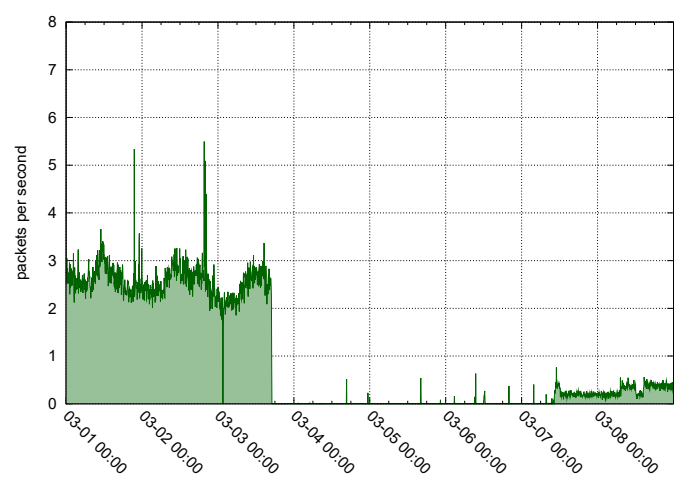

Figure 16: UCSD darknet's traffic coming from Libya: detail of the third outage. The small but visible amount of traffic during the third outage (coming from a small number of $/ 24$ networks) is consistent with the use of selective packet filtering, instead of BGP withdrawals, to effect the outage. 
ages were BGP-based may indicate that the censors were unsatisfied with the results of these tests, and used BGP-based disruption for the first two outages as an alternative while they further tested packet filtering techniques.

\subsubsection{Denial of service attacks}

In addition to reflecting the outages, our analysis of the UCSD darknet traffic shows two denial-of-service attacks to systems located in Libya. Because these attacks used randomly spoofed source addresses, we do not know if the attackers were inside or outside the country (or both), or how many machines were used to source the attack. The first attack, labeled D1 in Figure 12, started on February 26 at 20:27 UTC, targeted a few IPs in a subnet of the Libyan Telecom (ltt.ly) and lasted approximately 24 hours and 19 minutes. Analysis of the backscatter traffic allows us to estimate an average packet rate of 30390 packets per second.

The second attack, labeled D2, started on Saturday March 19 2011 at 20:31 UTC and the victim was a single IP assigned to the Libyan telecom provider. The attack lasted for about 27 hours and 51 minutes with an estimated average packet rate of 30280 packets per second.

\section{DISCUSSION AND CONCLUSION}

Political events in the Middle East this year, as well as political discussions in the U.S. Congress [55] have inspired popular as well as technical interest in possible mechanisms, impact, circumvention, and detection of Internet filtering at different layers. Our study of Egypt's and Libya's government-ordered Internet outages have revealed number of challenges and opportunities for the scientific study of Internet filtering and disruption. Given the growing interest and expanding circumstances that will give rise to large-scale Internet filtering behaviors, and the need to inform policy development with the best available empirical data and analysis of such behavior, we believe the topic will necessarily merit its own discipline. This study offers an intitial contribution in this direction.

We used multiple types of large-scale data in this analysis, all from data sets already available to academic researchers. The first type of data - BGP interdomain routing control plane data - was already widely analyzed and reported on during the outages. Our analysis of BGP data suggested that key decisions related to the outage were quite synchronized, and produced dramatic, globally observable consequences.

The second type of data - unsolicited data plane traffic to unassigned address space (darknet or telescope data) - we have not seen previously used for this purpose, and we were surprised at the range of insights it yielded. Unsolicited and unwanted traffic on the Internet has grown to such significant levels that instrumentation capturing such traffic can illuminate many different types of macroscopic events, including but not limited to broadscale packet-filtering-based censorship, which is not observable in BGP data. From this unidirectional traffic data we detected what we believe were Libya's attempts to test firewall-based blocking before they executed more aggressive BGP-based disconnection. This data also revealed Libya's use of such packet filtering technology during the second BGP-based connection. Interestingly, the backscatter component of this traffic data enabled us to identify some denial-of-service attacks against Egyptian government web sites before and after the censorship interval.

We also made limited use of active ping and macroscopic traceroute measurements toward address space in these countries during the outages. We used CAIDA's IPv4 topology data set to observe surprising two-way connectivity surviving the outage intervals that span more then a day. Using both control plane and data plane data sets in combination allowed us to narrow down which form of Internet access disruption was implemented at different times in a given region.

Our methodology required determining which IP address prefixes were in each country using RIR-delegation data and public geolocation database (MaxMind) data, and then mapping those prefixes of interest to origin ASes using publicly available BGP data repositories in the U.S. and Europe. Looking deeper into all sources of data can reveal different filtering approaches, possible satellite jamming, ranges of IPs not filtered by the firewall; different forms of (or lack of) coordination with other authorities. These techniques could also be used to improve the accuracy of geolocation databases, e.g., detecting errors in geolocation databases that map IP addresses to completely censored countries, but such IP addresses still show up in measurements.

We also note that BGP-based and firewall-based traffic control are only two of many layers where connectivity and content censorship may occur. A taxonomy of blocking technologies would include everything from physical layer disconnection, DPI-based content blocking by an ISP, DNS-based blocking or manipulation at multiple granularities, and end-client software blocking in an enterprise or household.

Since IPv6 is not as widely deployed as IPv4, there is a lack of feature parity in IPv4 and IPv6 technologies, including censorship technologies such as deep packet inspection. This disparity means that IPv6 may offer a time-limited opportunity for evasion of layer3 IP censorship. The fact that all IPv6 prefixes in Egypt were unaffected by the outage, shows that currently data flows using IPv6 are easily overlooked or ignored. Whether it was a willful act of disobedience by the network operators or (more likely) an oversight, the effect is the same: unless or until IPv6 gains considerably more traction, IPv6 data flows may remain "under the radar" (or more accurately, may continue to slip through the firewall).

Additional data sources would deepen the analysis, especially since only a subset of data sources may be available at any time. We used Ark data as supporting evidence for bidirectional layer 3 reachability of prefixes in Egypt and Libya, but the same data also contains forward path information that we have not yet explored. This data should allow us to see path changes at the data plane that happened due to the censorship we described. Comparing path changes observed in the data plane with those observed in the control plane, i.e. BGP, may expose non-BGP routing phenomena, such as use of default routes in the default free zone [13].

The opportunity to observe such a macroscopic event is a historical experience, and would not be possible without public (or at least researcher) access to globally relevant Internet data archives. Indeed, additional sources of data would further improve our ability to characterize the nature, timing, and effects of outages. A realtime hybrid BGP+traceroute+traffic monitoring system with geolocation capability would be able to capture such events in far greater detail. The methodology we developed could be used, augmented, and automated, to detect outages or similar macroscopically disruptive events in other geographic or topological regions. Our next step is to expand our use of CAIDA's macroscopic topology data for this purpose, as well as using Ark's measurement-on-request functionality to trigger higher fidelity measurements to observably affected areas, similar to what the Hubble project does for singleprefix outages [29].

\section{Acknowledgements}

We are grateful to the anonymous reviewers for their feedback. We would also like to thank Niels Raijer for assistance in understanding satellite technology. This material is based upon work supported by 
the National Science Foundation under Grant No. 1059439. Antonio Pescapé has been partially supported by LINCE project of the FARO programme jointly financed by the Compagnia di San Paolo and by the Polo delle Scienze e delle Tecnologie of the University of Napoli Federico II. Support for the UCSD network telescope operations and data collection, curation, analysis, and sharing is provided by DHS S\&T NBCHC070133 and UCSD. The Ark infrastructure operations and data collection, curation, and sharing, as well as Kimberly Claffy's effort on this project, is supported by DHS S\&T NBCHC070133, DHS S\&T N66001-08-C-2029, and NSF CNS-0958547. Emile Aben was supported by RIPE NCC, although the majority of his contribution was on his own time so as to not interfere with his RIPE-NCC responsibilites. Marco Chiesa and Claudio Squarcella were partially supported by MIUR of Italy under project AlgoDEEP prot. 2008TFBWL4. Part of their research was conducted in the framework of ESF project 10-EuroGIGA-OP003 GraDR "Graph Drawings and Representations".

\section{REFERENCES}

[1] AfriNIC: The Registry of Internet Number Resources for Africa. http://www.afrinic.net.

[2] Archipelago Measurement Infrastructure. http://www . caida.org/projects/ark.

[3] Opennet initialive. http://opennet.net.

[4] RIPE NCC: BGPviz. http://www.ris.ripe.net/bgpviz/.

[5] RIPE NCC: REX, Resources EXplained. http://albatross.ripe.net/cgi-bin/rex.pl.

[6] RIPE NCC: Routing Information Service (RIS). http://www.ripe.net/datatools/stats/ris/routing-information-service.

[7] UCSD Network Telescope, 2010. http://www . caida. org/data/passive/network_telescope.xml.

[8] Freedom on the Net 2011: A Global Assessment of Internet and Digital Media. http://www. freedomhouse.org/ images/File/FotN/FOTN2011.pdf, Apr. 2011.

[9] E. Aben. Conficker/Conflicker/Downadup as seen from the UCSD Network Telescope. http: //www . caida.org/research/security/ms08067/conficker. xml, 2008.

[10] E. Aben. Unsolicited Internet Traffic from Libya. http://labs.ripe.net/Members/emileaben/ unsolicited-internet-traffic-from-libya, March 2011.

[11] BGPmon. Internet in Egypt offline. http://bgpmon. net/blog/?p=450, January 2011.

[12] J. Burris. Comcast: Internet service restored after regional outage. http://articles.baltimoresun. com/201011-29/news/bs-md-comcast-20101128_1_outageinternet-service-disruptions, Nov 292010.

[13] R. Bush, O. Maennel, M. Roughan, and S. Uhlig. Internet optometry: assessing the broken glasses in Internet reachability. In ACM SIGCOMM conference on Internet measurement, 2009.

[14] R. Clayton, S. Murdoch, and R. Watson. Ignoring the Great Firewall of China. Privacy Enhancing Technologies workshop 2006, 4258:20-35, June 2006.

[15] J. Cowie. Strange Changes in Iranian Transit. http://www.renesys. com/blog/2009/06/strangechanges-in-iranian-int.shtml, Jun 142009.

[16] J. Cowie. Egypt Leaves the Internet. http: //www.renesys.com/blog/2011/01/egyptleaves-the-internet.shtml, Jan 272011.
[17] J. Cowie. Libyan Disconnect. http://www.renesys.com/blog/2011/02/libyandisconnect-1. shtml, February 2011.

[18] J. Cowie. What Libya Learned from Egypt. http: //www.renesys.com/blog/2011/03/whatlibya-learned-from-egypt. shtml, March 2011.

[19] J. Cowie, A. Ogielski, B. Premore, E. Smith, and T. Underwood. Impact of the 2003 blackouts on Internet communications. November 2003.

[20] J. Cowie, A. Ogielski, B. Premore, and Y. Yuan. Internet worms and global routing instabilities. In Proc. of SPIE International symposium on Convergence of IT and Communication, July 2002.

[21] J. Crandall, D. Zinn, M. Byrd, E. Barr, and R. East. ConceptDoppler: A Weather Tracker for Internet Censorship. In Proceedings of the 14th ACM conference on Computer and communications security, pages 352 - 365, 2007.

[22] A. Dainotti, A. Pescapé, and G. Ventre. Worm traffic analysis and characterization. In IEEE ICC 2007, June 2007.

[23] S. Deshpande, M. Thottan, T. K. Ho, and B. Sikdar. An Online Mechanism for BGP Instability Detection and Analysis. IEEE Trans. Comput., 58:1470-1484, November 2009.

[24] S. Garret. We can confirm that Twitter was blocked in Egypt around 8am PT today.... http://twitter.com/\#! /twitterglobalpr/status/30063209247408128, Jan 252011.

[25] Google. Google Transparency Report. http://www . google.com/transparencyreport/traffic/?r=LY\&l= YOUTUBE\&csd $=1296723600000 \& c e d=1299142800000$.

[26] Google. Google Transparency Report. http://www . google.com/transparencyreport/traffic/?r=LY\&l= EVERYTHING\&csd=1296862200000\&ced $=1299281400000$.

[27] B. Huffaker, M. Fomenkov, and kc claffy. Geocompare: a comparison of public and commercial geolocation databases. In Network Mapping and Measurement Conference (NMMC), May 2011. http://www . caida.org/ publications/papers/2011/geocompare-tr/.

[28] Y. Hyun, B. Huffaker, D. Andersen, E. Aben, C. Shannon, M. Luckie, and K. C. Claffy. The IPv4 Routed /24 Topology Dataset, 2011. http: //www . caida.org/data/active/ ipv4_routed_24_topology_dataset.xml.

[29] E. Katz-Bassett, H. V. Madhyastha, J. P. John, A. Krishnamurthy, D. Wetherall, and T. Anderson. Studying Black Holes in the Internet with Hubble. USENIX Symposium on Networked Systems Design \& Implementation (NSDI), 2008.

[30] R. Khosla, S. Fahmy, and Y. C. Hu. BGP Molecules: Understanding and Predicting Prefix Failures. In Proc. IEEE INFOCOM Mini-Conference 2011, 2011.

[31] C. Labovitz. Libya Firewall Begins to Crumble? http://monkey.org/ labovit/blog//viewpage .php? page=libya_firewall_cracks.

[32] C. Labovitz. Egypt Loses the Internet. http://monkey.org/ labovit/blog/viewpage.php? page=egypt_loses_internet, Jan 282011.

[33] C. Labovitz. Egypt returns to the Internet. http://asert . arbornetworks. com/2011/02/egyptreturns-to-the-internet/, February 2011.

[34] C. Labovitz. Middle East Internet Scorecard. http: //asert. arbornetworks. com/2011/02/middle-eastinternet-scorecard-february-12-\%E2\%80\%93-20/, February 2011. 
[35] C. Labovitz, G. R. Malan, and F. Jahanian. Origins of Internet Routing Instability. In Proc. IEEE INFOCOM, pages 218-226, 1999.

[36] C. Labovitz, S. Member, G. R. Malan, S. Member, and F. Jahanian. Internet routing instability. In IEEE/ACM Transactions on Networking, pages 515-528, 1997.

[37] S. LaPerriere. Taiwan earthquake fiber cuts: a service provider view. NANOG 39, February 2007.

[38] J. Li and S. Brooks. I-seismograph: Observing and measuring Internet earthquakes. Infocom 2011 proceedings, April 2011.

[39] G. Mahlknecht. Greg's Cable Map. http://www. cablemap.info/, retrieved 12 September 2011.

[40] MaxMind. MaxMind GeoLite Country: Open Source IP Address to Country Database. http://www .maxmind.com/app/geolitecountry.

[41] Ministry of Communications and Information Technology, Arab Republic of Egypt. ICT indicators in brief. http://www.mcit.gov.eg/Upcont/Documents/ ICTinBriefFeb2011-E. pdf, Feb 2011.

[42] D. Moore, V. Paxson, S. Savage, C. Shannon, S. Staniford, and N. Weaver. Inside the Slammer Worm. IEEE Security and Privacy, 1(4):33-39, July 2003.

[43] D. Moore, C. Shannon, D. J. Brown, G. M. Voelker, and S. Savage. Inferring Internet denial-of-service activity. ACM Trans. Comput. Syst., 24:115-139, May 2006.

[44] S. Nasrawi. Libya Protests: Anti-Government Protesters Killed During Day Of Rage. http://www . huffingtonpost . com/2011/02/17/ libya-protests-antigovern_0_n_824826.html, February 2011.

[45] R. Pang, V. Yegneswaran, P. Barford, V. Paxson, and L. Peterson. Characteristics of Internet background radiation. In Proceedings of the 4th ACM SIGCOMM conference on Internet measurement, IMC '04, pages 27-40, New York, NY, USA, 2004. ACM.

[46] I. Poese, S. Uhlig, M. A. Kaafar, B. Donnet, and B. Gueye. IP Geolocation Databases: Unreliable? ACM SIGCOMM Computer Communication Review, 41(2), April 2011.

[47] Reuters. http://af.reuters.com/article/ libyaNews/idAFLDE71N2CU20110224, February 2011.

[48] Reuters. Egypt govt denies disrupting websites - cabinet. http://www.reuters.com/article/2011/01/26/ egypt-web-idUSLDE70P28720110126, Jan 262011.

[49] Reuters. Facebook says has seen drop in traffic from Egypt. http://www.reuters.com/article/2011/01/27/ facebook-egypt-idUSN2727880720110127, Jan 2011.

[50] RIPE. Analysis of Egyptian Internet outage 27th January 2nd February 2011. http://stat.ripe.net/egypt, 2011.

[51] A. Sahoo, K. Kant, and P. Mohapatra. Characterization of BGP Recovery Time under Large-Scale Failures. In IEEE International Conference on Communications, volume 2, 2006.

[52] Y. Shavitt and N. Zilberman. A study of geolocation databases. Journal on Selected Areas of Communications, abs/1005.5674, 2011.

[53] M. Skoric, N. Poor, Y. Liao, and S. Tang. Online Organization of an Offline Protest: From Social to Traditional Media and Back. In System Sciences (HICSS), 2011 44th Hawaii International Conference on, pages 1 -8, jan. 2011.

[54] T. D. Star. http://www.dailystar.com.1b/News/
Politics/Feb/23/Libya-source-of-jamming-ofLebanese-news-channels-TRA . ashx\#axzz1Ev3JP000, February 2011.

[55] J. Swartz. 'Kill Switch' Internet bill alarms privacy experts. http://www.usatoday.com/tech/news/ internetprivacy/2011-02-15-kill-switch_N.htm, Feb 152011

[56] S. T. Teoh, S. Ranjan, A. Nucci, and C.-N. Chuah. BGP eye: a new visualization tool for real-time detection and analysis of BGP anomalies. In Proceedings of the 3rd international workshop on Visualization for computer security, VizSEC '06, pages 81-90, New York, NY, USA, 2006. ACM.

[57] T. Underwood. Con-Ed Steals the 'Net. http: //www.renesys.com/blog/2006/01/coned_ steals_the_net.shtml, Jan 222006.

[58] R. T. University. BGPlay - graphical visualisation of BGP updates. http://bgplay.routeviews.org/.

[59] University of Oregon. University of Oregon Route Views project. http://www . routeviews . org.

[60] I. van Beijnum. How Egypt did (and your government could) shut down the Internet. http://arstechnica.com/techpolicy/news/2011/01/how-egypt-or-how-yourgovernment-could-shut-down-the-internet.ars, February 2011.

[61] T. Wan and P. van Oorschot. Analysis of BGP prefix origins during Google's May 2005 outage. In Parallel and Distributed Processing Symposium, 2006. IPDPS 2006. 20th International, page 8 pp., april 2006.

[62] Wikipedia. Ramses Exchange. http://en.wikipedia.org/wiki/Ramses_Exchange, Sep 122011.

[63] B. Woodcock. Overview of the Egyptian Internet Shutdown. http://www.pch.net/resources/misc/Egypt-PCHOverview .pdf, Feb 2011.

[64] X. Xu, Z. M. Mao, and J. A. Halderman. Internet Censorship in China: Where Does the Filtering Occur? Proceedings of PAM 2011, 2011.

[65] H. Yan, R. Oliveira, K. Burnett, D. Matthews, L. Zhang, and D. Massey. BGPmon: A real-time, scalable, extensible monitoring system. In Proceedings of IEEE Conference on Cybersecurity Applications and Technologies Conference for Homeland Security, 2009.

[66] E. Zmijewski. Georgia Clings to the 'Net. http://www . renesys. com/blog/2008/08/georgiaclings-to-the-net.shtml, Aug 102008.

[67] E. Zmijewski. Mediterranean cable break. http://www.renesys.com/blog/2008/01/ mediterranean_cable_break.shtml, Jan 302008.

[68] E. Zmijewski. Mediterranean cable break - part ii. http://www.renesys.com/blog/2008/01/ mediterranean_cable_break_part_1.shtml, Jan 31 2008.

[69] E. Zmijewski. Mediterranean cable break - part iii. http://www.renesys.com/blog/2008/02/ mediterranean_cable_break_part.shtml, Feb 22008.

\section{APPENDIX}

\section{A. ETHICAL CONSIDERATIONS}

We recognize that this paper could possibly expose network operators' disobedience to government orders, with potential harmful consequences to the operators. Therefore we believe it would be 
ethical to anonymize the ASes that might be at risk for harm. Here we describe and justify the reasoning for our anonymization policy.

Our main concern is that we do not know the nature of the relationships between the operators of these networks to their governments, whether they disobeyed direct orders and what the penalties might be. We assume that the national incumbent telecom operators will follow orders from their respective governments; our research did not reveal behavior that suggests otherwise. Furthermore, the behavior of both incumbent telecom operators has been described in detail elsewhere, e.g., [16]. So in this paper we label these ASes EgStateAs and LyStateAs. For the remaining ASes we anonymized ASes that were operating nationally in Egypt with an Eg-prefix, e.g., EgAs1, EgAs2, etc. We did not need to do so for Libya, because we didn't find ASes operating at the national level in Libya, except for the state-owned telecom operator.
As the upstreams of networks in Libya and Egypt may have personnel operating in one of these countries, we decided to anonymize these as well, and labeled them IntAs1, IntAs 2, etc. One distinguishing characteristic of censorship activity we observed is whether it occurs via wireless satellite connectivity; depending on who owns the satellite links, disrupting such connectivity may require jamming technology rather than firewall-based filtering. To acknowledge this distinction, which is revealed in the data anyway, we labeled the one international satellite connectivity provider we show data for SatAs1. We realize this anonymization policy cannot guarantee to prevent deanonymization by careful examination of publicly available data, but it raises the threshold for doing so without compromising the scientific value of this paper. 